Objectives: This Phase 2 study was designed to minimize background medications and placebo responses to improve interpretation of a small trial in a complex, heterogenous disease.

Methods: SLE patients were enrolled with active disease, ameliorated during screening with $\geq 160 \mathrm{mg}$ of IM Depo-Medrol. Improvement was required before randomization, defined by decrease in SLEDAI $>4$ points or $>1$ grade in a BILAG A or B score. Immunosuppressive drugs were stopped except antimalarials and/ or $\leq 10 \mathrm{mg} /$ day prednisone or equivalent. Subjects were randomized to IV $\mathrm{XmAb5871}$ (5 mg/kg) or placebo and given Depo-Medrol $80 \mathrm{mg}$ IM on Days 1 and 15 , after which, steroid impact was expected to withdraw gradually. Study treatments were given Q14 days for up to 16 doses or loss of improvement (LOI), defined as SLEDAI increase $\geq 4$ points OR new BILAG A or B, with investigatordetermined significance. At LOI, patients could receive immediate standard treatments. The primary endpoint was the proportion with no LOI by Day 225 in the efficacy evaluable group (those completing Day 225 or withdrawn for LOI or drugrelated adverse event).

Results: 104 subjects were randomized: 99 female, median age 45 (2065). The primary endpoint was met by 21 (42\%) of XmAb5871-treated patients vs $12(28.6 \%)$ of the placebo group $(p=0.18)$. All but one responder also fulfilled the SRI-4 response definition from screening to completion. Results did not differ in those with or without anti-dsDNA and/or ENA antibodies. Time to flare was significantly longer in the XmAb5871 group $(p=0.025)$ (figure 1). XmAb5871-treated patients with LOI had less recurrent disease after $\mathrm{IM}$ steroid cessation than those in the placebo group; 6 (20\%) of placebo patients developed BILAG A scores vs 3 $(13 \%)$ in the active arm. $9(30 \%)$ of worsening placebo patients had SLEDAI increase $\geq 7$ vs 0 in the XmAb5871 group. SLEDAI scores were higher and increased sooner after disease nadir with placebo vs XmAb5871 (figure 2); 16 (30.8\%) of XmAb5871 patients vs 7 (13.5\%) placebo patients sustained LLDAS (low disease) during months 6-8 $(p=0.0453)$. Transient, infusion-related gastrointestinal side effects occurred in XmAb5871-treated patients during the 1 st or $2^{\text {nd }}$ infusion. There were 8 SAEs in 7 XmAb5871-treated subjects, 5 in 4 placebo patients, no opportunistic infections, and no deaths. Infection rate was low compared to other SLE trials.

Conclusion: XmAb5871 was well-tolerated. Preliminary data from this small trial indicates suppression of disease recurrence after treatment withdrawal, supporting further evaluation of XmAb5871 in SLE.

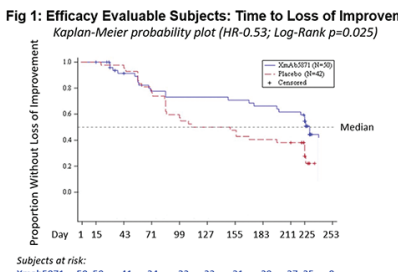

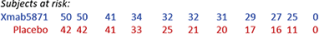

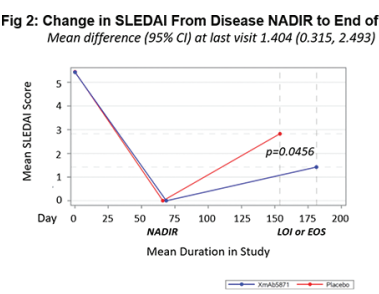

Disclosure of Interests: Joan Merrill Grant/research support from: Genentech, UCB, GSK, EMD Serono, Pfizer, Celgene, Exagen, Bristol Myers Squibb, Medimmune/Astra Zeneca, Lilly, Amgen, Xencor, Neovacs, Consultant for: Genentech, UCB, GSK, EMD Serono, Pfizer, RemeGen, Celgene, Exagen, Bristol Myers Squibb, Medimmune/Astra Zeneca, Lilly, Immupharma, Amgen, Janssen, Sanofi, Neovacs, Anthera, Speakers bureau: UCB, GSK, EMD Serono, Bristol Myers Squibb, Medimmune/Astra Zeneca, Janssen, Joshua June: None declared, Fotios Koumpouras: None declared, Wambui Machua: None declared, Mohammad Faisal Khan: None declared, Anca Askanase: None declared, Arezou Khosroshahi: None declared, Saira Sheikh: None declared, Judith A. James: None declared, Joel Guthridge: None declared, Gaurav Rathi Shareholder of: Xencor Inc, Employee of: Xencor Inc, Bart Burington Shareholder of: Xencor Inc, Employee of: Xencor Inc, Paul Foster Shareholder of: Xencor Inc, Employee of: Xencor Inc, Debra Zack Shareholder of: Xencor Inc, Employee of: Xencor Inc

DOI: 10.1136/annrheumdis-2019-eular.4234

\section{FRI0177 \\ THE ADDITIVE EFFECTS OF HYDROXYCHLOROQUINE TO MAINTENANCE THERAPY WITH STANDARD OF CARE IN PATIENTS WITH SYSTEMIC LUPUS: ERYTHEMATOSUS}

Ippei Miyagawa, Kazuhisa Nakano, Shingo Nakayamada, Shigeru Iwata,

Kentaro Hanami, Shunsuke Fukuyo, Satoshi Kubo, Akio Kawabe,

Yusuke Miyazaki, Yoshino Inoue, Masanobu Ueno, Yoshiya Tanaka. University of Occupational and Environmental Health, Japan, The First Department of Internal

Medicine, Kitakyusyu, Japan

Background: Antimalarial agents such as hydroxychloroquine (HCQ) have long been used as effective therapies for skin and joint symptoms, as well as for the malaise associated with cutaneous lupus erythematosus and systemic lupus erythematosus (SLE). Furthermore, based on the various benefits demonstrated with these agents, the use of antimalarial agents was recently recommended for all patients with SLE. Whereas HCQ has been generally given to most of patients from the beginning of the treatment during the remission-induction therapy in multiple studies, its effects on maintenance therapy have not been sufficiently supported by evidence.

Objectives: We evaluated the additive effects of $\mathrm{HCQ}$ in maintenance therapy with standard of care (SoC) in 101 patients with SLE for 1 year. Methods: The study included 101 patients diagnosed with SLE, whose course was followed for 1 year at our hospital and affiliated institutions. All patients were receiving maintenance therapy based on the SoC. The primary endpoint was the changes in the SLEDAI. The secondary endpoints were the proportion of emergence of new BILAG $A$ or $B$ organ domain score, and changes in anti-ds DNA Ab titre $(U / \mathrm{mL})$, and serum complement activity $(\mathrm{CH} 50, \mathrm{U} / \mathrm{mL})$ up to year 1 , as well as the CS-sparing effect. For these endpoints, the $\mathrm{SoC}_{+} \mathrm{HCQ}$ group $(\mathrm{n}=42)$ was compared with the SoC group $(n=59)$ of patients matched for baseline characteristics. The Human Ethics Review Committee of our university reviewed and approved this study.

Results: In the $\mathrm{SoC}+\mathrm{HCQ}$ group, the mean age was 42.2 years, and there were 3 male and 39 female. The mean disease duration was 157.9 months. In the SoC group, the mean age was 43.5 years, and there were 6 male and 53 female. The mean disease duration was 118.9 months. At baseline, no statistically significant differences between the two groups were observed in any baseline characteristics. The SLE DAl improved from 3.07 to 2.28 in the $\mathrm{SoC}+\mathrm{HCQ}$ group, but significantly deteriorated from 2.73 to 4.8 in the $\mathrm{SoC}$ group. The $\mathrm{CH} 50$ levels, antidsDNA antibody titre, and concomitant CS dose were not significantly changed. The increase in the SLEDAI and concomitant CS dose after 1 year were all significantly greater in the SoC group, and the proportion of patients with SLEDAI flare was significantly lower in the SoC+HCQ group $(4.76 \%$ vs $25.4 \%$ ) (SLEDAl flare was defined as an increase of at least four points in the SLEDAI). Regarding the BILAG organ domain, there were no significant differences. SLEDAI flare were observed in 17 patients. When baseline characteristics were compared between patients with and without SLEDAI flare, $\mathrm{HCQ}$ was significantly more frequently used in patients without SLEDAI flare. In addition, univariate and multivariate logistic regression analyses were performed to identify the predictive factors for no SLEDAI flare. The univariate logistic analysis identified $\mathrm{HCQ}$ use, and immunosuppressant use with a $\mathrm{P}$ value of $<0.3$. Subsequently, multivariate logistic analysis was performed with these factors as dependent variables and identified the presence or absence of $\mathrm{HCQ}$ use as a predictive factor $(P=0.0041$, odds ratio $6.66,95 \%$ confidence interval 1.73-44.1). The retention rate of $\mathrm{HCQ}$ was $90.5 \%$.

Conclusion: The comparison between the $\mathrm{SoC}+\mathrm{HCQ}$ and $\mathrm{SoC}$ groups revealed that the addition of $\mathrm{HCQ}$ to maintenance therapy with low-dose CS for SLE is safe, and that HCQ was effective, not only for the suppression of disease activity based on the SLEDAI, but also for the prevention of the exacerbation of disease activity. Thus, the present study revealed that $\mathrm{HCQ}$ may be a useful mainstay for maintenance therapy based on SoC in patients with SLE.

Disclosure of Interests: Ippei Miyagawa: None declared, Kazuhisa Nakano: None declared, Shingo Nakayamada Grant/research support from: Mitsubishi-Tanabe, Takeda, Novartis and MSD, Speakers bureau: BristolMyers, Sanofi, Abbvie, Eisai, Eli Lilly, Chugai, Asahi-kasei and Pfizer, Shigeru Iwata: None declared, Kentaro Hanami: None declared, Shunsuke Fukuyo: None declared, Satoshi Kubo Speakers bureau: Bristol-Myers, Pfizer, Takeda, and Eli Lilly, Akio Kawabe: None declared, Yusuke Miyazaki: None declared, Yoshino Inoue: None declared, Masanobu Ueno: None declared, Yoshiya Tanaka Grant/research support from: Abbvie, Astellas, Bristol-Myers Squibb, Chugai, Daiichi-Sankyo, Eisai, MitsubishiTanabe, MSD, Ono, Taisho-Toyama, Takeda, Speakers bureau: Abbvie, Asahi-kasei, Astellas, Bristol-Myers Squibb, Chugai, Daiichi-Sankyo, Eli Lilly, Eisai, Glaxo-Smithkline, Janssen, Mitsubishi-Tanabe, Novartis, Pfizer Japan Inc, Sanofi, Takeda, UCB, YL Biologics 


\section{FRI0178 CLUSTER PROFILING OF PATIENTS IN A REAL-WORLD DATA SET WITH SYSTEMIC LUPUS ERYTHEMATOSUS AND THEIR ASSOCIATED TREATMENTS}

Zahi Touma ${ }^{1}$, Ben Hoskin ${ }^{2}$, Christian Atkinson ${ }^{2}$, David Bell², Olivia Massey², Jennifer H. Lofland ${ }^{3}$, Pam Berry ${ }^{4}$, Chetan Karyekar ${ }^{3}$, Karen Costenbader ${ }^{5}$. ${ }^{1}$ University of Toronto, Toronto, Canada; ${ }^{2}$ Adelphi Real World, Cheshire, United Kingdom; ${ }^{3}$ Janssen Global Commercial Strategic Organisation, Horsham, United States of America; ${ }^{4}$ Janssen Global Services, LLC, Horsham, United States of America; ${ }^{5}$ Brigham and Women's Hospital, Boston, United States of America

Background: Previous systemic lupus erythematosus (SLE) studies have identified potential clusters of SLE clinical manifestations.

Objectives: To describe the presentation of SLE across different cohorts of patients and describe standard of care within clusters.

Methods: Cross-sectional study of 263 rheumatologists in the US and EU5. Data were collected from the Adelphi Real World 2015 Lupus Disease Specific Programme. Physicians completed patient record forms (PRFs) for the next 5 patients consulting with SLE; these patients completed patient self-completion (PSC) forms describing how SLE affected them. PRFs data include patient's characteristics and management history. PSCs focused on similar data collection, including patient reported outcome measures on the humanistic burden. Principal-component factor analysis reduced 39 unique SLE symptoms to 8 factors. These factors were used as covariates in latent class cluster analysis to provide discrete cohorts of patients. Chi-squared and Kruskal-Wallis tests compared patient outcomes across clusters.

Results: Data were extracted from 1376 PRFs. Factor analysis resulted in 8 clusters of concurrent symptoms; joint, haematological, constitutional/ mental health, skin, circulatory, cardiovascular, renal, and muscular symptoms respectively. The four-cluster solution was selected.

Cluster 1 displayed the lowest symptom burden, characterised by low skin involvement. Cluster 2 is characterised by joint and skin involvement. Cluster 3 \& 4 had a high frequency of all factors, with cardiovascular involvement high in cluster 3 and renal/constitutional involvement high in cluster 4 (table 1).

Significant between-cluster differences were observed when comparing clinical and humanistic outcomes; physician/patient satisfaction were greatest in cluster 1 (physician satisfied $94.2 \%$ vs. 2: $90.8 \%, 3: 85.2 \%, 4$ : $74.4 \%, \quad p<0.0001$; patient $94.7 \%$ vs. 2: $93.9 \%, 3: 91.5 \%, 4: 79.2 \%$, $\mathrm{p}<0.0001$ ), whilst disease progression (deteriorating slowly $2.5 \%$ vs. 2 : $12.9 \%, 3: 9.8 \%, 4: 25.5 \%, p<0.0001)$ and flaring in the last 12 months (flared $30.0 \%$ vs. 2: $54.8 \%, 3: 62.2 \%, 4: 70.8 \%, p<0.0001$ ) differed significantly with worst outcomes seen in cluster 4 .

Significant differences were also observed between clusters in relation to treatment proportions; anti-malarials (highest cluster 1: 70.5\%), biologic DMARD (highest cluster 3: 17.5\%), glucocorticoid and immunosuppressants (highest cluster 4: $85.5 \%, 74.5 \%$ ).

Conclusion: This study adds to the evidence demonstrating the heterogenous nature of SLE experienced within distinct patient clusters. Significant proportions of SLE patients experience high symptom burden and low levels of satisfaction. Additional analysis to understand limited biologic use in more severe patients is needed.

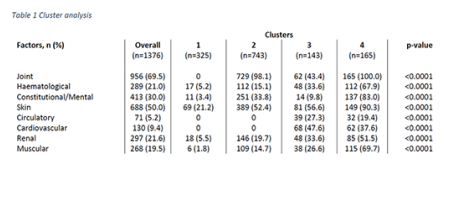

Disclosure of Interests: Zahi Touma Grant/research support from: GSK Canada, Consultant for: UBC, Pfizer, Janssen, Inc, Ben Hoskin Employee of: Adelphi, Christian Atkinson Employee of: Adelphi, David Bell Employee of: Adelphi, Olivia Massey Employee of: Adelphi Real World, Jennifer H. Lofland Employee of: Janssen Global Commercial Strategic Organization, Pam Berry Shareholder of: GSK and Janssen Global Services, Chetan Karyekar Shareholder of: J\&J, Employee of: Janssen Scientific Affairs, LLC, Abbott, BMS, Novartis, Karen Costenbader: None declared DOI: 10.1136/annrheumdis-2019-eular.4302

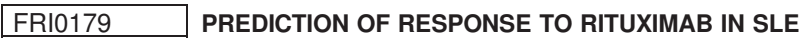 USING A VALIDATED TWO-SCORE SYSTEM FOR INTERFERON}

Adewonuola Alase $^{1}$, Zoe Wigston ${ }^{1}$, Agata Burska ${ }^{1}$, Elizabeth Hensor ${ }^{1}$, M.D. Yuzaiful M.D. Yusof ${ }^{1}$, John Reynolds ${ }^{2}$, The Masterplans Consortium ${ }^{3}$, Miriam Wittmann ${ }^{1}$, Ian N. Bruce ${ }^{2}$, Edward Vital ${ }^{1} .{ }^{1}$ Leeds Institute of Rheumatic and Musculoskeletal Medicine, Leeds, United Kingdom; ${ }^{2}$ University of Manchester, Centre for Musculoskeletal Research, Division of Musculoskeletal and Dermatological Sciences, Manchester, United Kingdom; ${ }^{3}$ University of Manchester, The MASTERPLANS project Team, Manchester, United Kingdom

Background: Rituximab (RTX) is used for resistant SLE but clinical response varies. We previously validated two interferon-stimulated gene expression scores (IFN-Score-A and IFN-Score-B) that improved prediction of clinical outcomes in SLE. IFN-Score-A included most commonly reported ISGs and predicted flares and glucocorticoid requirements. IFNScore-B included ISGs that respond to multiple IFN subtypes and predicted development of SLE in At-Risk individuals. Diagnosis of SLE was associated with both scores, while only IFN-Score-B was elevated in RA The British Society for Rheumatology Biologics Registry (BILAG-BR) collects samples for RTX-treated patients in the UK. MASTERPLANS is a consortium to identify predictors of drug response.

Objectives: To investigate whether IFN-Score-A and IFN-Score-B predict BILAG response to RTX at 6 months.

Methods: This is a preliminary analysis of the first RTX-treated patients in the BILAG-BR with complete data. Patients were recruited if they were starting a first cycle of RTX for active SLE (BILAG A or 2xBILAG B) despite previous cyclophosphamide or mycophenolate mofetil. Disease activity was measured using BILAG-2004. Clinical response was defined as improvement by $>=1$ grade in active BILAG-2004 systems with no worsening in other systems. Whole blood was collected into TEMPUS tubes and RNA extracted. IFN-Scores were measured using a custom Taqman array as previously described [El Sherbiny et al., 2018]. Multivariate logistic regression was used to test IFN-Scores and baseline clinical covariates as predictors of BILAG response at 6 months.

Results: Samples were available from 147 patients, of whom 84 had complete baseline and 6 month clinical data available and were included in this analysis. $40 / 84(47.6 \%)$ patients had BILAG response at 6 months. In univariate and multivariate analysis, high IFN-Score-B expression was significantly associated with clinical response (see table 1).

\begin{tabular}{lcccc}
\hline Predictor & $\begin{array}{c}\text { Non- } \\
\text { responders }\end{array}$ & Responders & Univariable OR (95\% & Multivariable OR (95\% \\
Cl)
\end{tabular}

